LBNL-62680

UCI-TR-2007-23

\title{
Bounds on Unparticles from the Higgs Sector
}

\author{
Patrick J. Fox ${ }^{a}$, Arvind Rajaraman ${ }^{b}$ and Yuri Shirman ${ }^{b}$ \\ ${ }^{a}$ Theoretical Physics Group, Lawrence Berkeley National Laboratory, Berkeley, CA 94720 \\ ${ }^{b}$ Department of Physics and Astronomy, University of California, Irvine, CA 92697
}

\begin{abstract}
We study supersymmetric QCD in the conformal window as a laboratory for unparticle physics, and analyze couplings between the unparticle sector and the Higgs sector. These couplings can lead to the unparticle sector being pushed away from its scale invariant fixed point. We show that this implies that low energy experiments will not be able to see unparticle physics, and the best hope of seeing unparticles is in high energy collider experiments such as the Tevatron and the LHC. We also demonstrate how the breaking of scale invariance could be observed at these experiments.
\end{abstract}




\section{Contents}

1. Introduction and Conclusions 1

2. Supersymmetric QCD as a model of unparticle physics 2

3. Operator analysis and experimental constraints 3

4. New effects in non-unparticle physics 5

\section{Introduction and Conclusions}

Recently there has been a lot of interest [1-8] in unparticle theories $[9,10]$ in which the Standard Model (SM) is coupled to a conformal sector (called the unparticle sector). As shown in $[9,10]$, the conformal sector can have interesting and unexpected consequences. In this note, we shall investigate the effects on the conformal sector from the Higgs sector, and we will show that this leads to surprising new bounds on unparticle physics.

The main point of our analysis is that the coupling to the Higgs sector is the most important operator; in fact, if there is a scalar unparticle operator of dimension less than 2, the coupling to the Higgs sector is through a relevant operator. When the Higgs gets a vacuum expectation value, this operator breaks the conformal invariance of the hidden sector. For unparticle physics to be relevant, this breaking scale is required to be sufficiently low. (This operator may also lead to strong effects on Higgs physics from unparticles, which would be interesting to investigate.)

This requirement imposes strong constraints on the unparticle sector. If these constraints are satisfied, low energy experiments will not be able to probe any aspects of unparticle physics. The only place where unparticle physics will be relevant is in high energy experiments like the Tevatron and the LHC, which can indeed probe unparticle physics. In fact, the breaking of conformal invariance may also be measurable as deviations from the predictions of unparticle physics.

We will begin by discussing a model of unparticle physics, which is different from the previously suggested models. We take the conformal sector to be a supersymmetric gauge theory, which at low energies flows to a conformal theory. Using supersymmetry, we will be able to explicitly calculate the dimensions of chiral operators in this theory, and show that this is a good model for unparticle (and now also unsparticle) physics. We note that anomalous dimensions in these theories can be large, in contrast to Banks-Zaks fixed points which are weakly coupled. 
We then couple this sector to the Standard Model, focusing on the Higgs sector. We show that conformal invariance is broken at low energies, and for reasonable choices of scales the inclusion of the Higgs-unparticle operator means that low energy experiments are unable to see the effects of unparticles. Finally, we propose a toy model for a theory with unparticles and a breaking of scale invariance, and calculate experimental predictions of this effect.

\section{Supersymmetric QCD as a model of unparticle physics}

We would like to have an example of a conformal field theory in which it is possible to do semi-quantitative calculations. This is challenging since we also require the theory be strongly coupled in order that anomalous dimensions can be large. Remarkably such an example exists in the literature [11,12], in the form of supersymmetric QCD (SQCD) in a certain regime. We will therefore consider SQCD in the conformal window as a laboratory for unparticle physics.

We briefly review the results of SQCD (for a comprehensive review see [13]). Consider SQCD with gauge group $S U\left(N_{C}\right)$ and $N_{F}$ vector-like quark superfields $(Q, \bar{Q})$ with $\frac{3}{2} N_{C}<$ $N_{F}<3 N_{C}$ (we call this the electric theory). Such a theory flows to a strongly coupled conformal fixed point in the infrared (IR). At the fixed point the theory has a dual (magnetic) description, with gauge group $S U\left(N_{F}-N_{C}\right), N_{F}$ dual-quark superfields $(q, \bar{q})$, a gauge singlet meson superfield $M$ (transforming in the bifundamental representation of the $S U\left(N_{F}\right) \times$ $S U\left(N_{F}\right)$ flavor symmetry, and superpotential,

$$
W_{m a g}=\bar{q} M q
$$

The meson of the magnetic description corresponds to the gauge invariant composite $\bar{Q} Q$ of the electric theory.

The magnetic conformal theory can now be coupled to the Standard Model, and will then be a candidate for the unparticle sector. In general, we can write the ultraviolet (UV) coupling of an operator of dimension $d_{U V}$ in the unparticle sector to a SM operator of dimension $l$ as

$$
\frac{1}{M_{\mathcal{U}}^{l+d_{U V}-4}} \mathcal{O}_{S M} \mathcal{O}_{U V}
$$

Below the strong coupling scale $\Lambda_{\mathcal{U}}$, these couplings flow to

$$
\mathcal{C} \frac{\Lambda_{\mathcal{U}}^{d_{U V}-d_{\mathcal{U}}}}{M_{\mathcal{U}}^{l+d_{U V}-4}} \mathcal{O}_{S M} \mathcal{O}_{I R}
$$

(in the notation of Georgi $[9,10] k=l+d_{U V}-4$ ).

Supersymmetric QCD allows us to make this explicit. For example, adding a superpotential coupling in the UV regime of the magnetic description

$$
W_{c}=\frac{1}{M_{\mathcal{U}}} H L \bar{e} \operatorname{Tr} M
$$


leads, among others, to the following terms in in the Lagrangian

$$
\frac{1}{M_{\mathcal{U}}} H L \bar{e} \operatorname{Tr} M+\left(\frac{1}{M_{\mathcal{U}}} H \tilde{L} \tilde{\bar{e}} q^{*} \bar{q}^{*}+\text { h.c. }\right) \text {, }
$$

which have the form (2.2) with $\mathcal{O}_{U V}=\operatorname{Tr} M$ of dimension $d_{U V}=1$ and $\mathcal{O}_{U V}=q \bar{q}$ of dimension $d_{U V}=2$. Below the strong coupling scale, once the theory reaches its conformal fixed point, the dimensions of these operators can be computed from their R-charges to be $d_{\mathcal{U}}=3 \frac{N_{C}-N F}{N_{F}}$ and $d_{\mathcal{U}}=3 \frac{N_{C}}{N_{F}}$ respectively. In the conformal window, the dimension of both operators lie between 1 and 2, making them perfect candidates for the operator $\mathcal{O}_{\mathcal{U}}$ of the unparticle conformal sector [9].

A couple of comments are in order:

1. For generic choices of $N_{C}$ and $N_{F}$, in the conformal window, the dimensions of the operators $q \bar{q}, M$ significantly differ from integer values. This is unlike the Banks-Zaks (BZ) theory [14] which has a weakly coupled fixed point where all operators have dimension close to their classical value. In particular, all gauge invariant operators in BZ theory have almost integer dimensions.

2. We can perturb the theory by adding a term to the action $\lambda \mathcal{O}_{I R}=\lambda \operatorname{Tr} M$. This corresponds to adding a mass term for the quarks of the electric description. The result of this mass term is that at low energies, the quarks can be integrated out, and the theory becomes a pure super-Yang Mills theory, which is no longer conformal.

\section{Operator analysis and experimental constraints}

The couplings of the unparticle sector and the SM sector can have interesting effects. Most interest thus far has concentrated on operators involving SM fermions and gauge bosons (with the goal of determining low energy signatures of unparticles) and consequently on operators with $l \geq 3$. Because the operator of lowest dimension in the unparticle sector has dimension greater than 1 , this means that the coupling operator is irrelevant $\left(l+d_{U V}-4 \geq 0\right)$.

However there is another type of coupling between the SM and the unparticle sector, involving the SM Higgs boson. The coupling is of the form

$$
\frac{1}{M_{\mathcal{U}}^{d_{U V}-2}}|H|^{2} \mathcal{O}_{U V}
$$

which flows in the infrared (IR) to

$$
C_{\mathcal{U}} \frac{\Lambda_{\mathcal{U}}^{d_{U V}-d_{\mathcal{U}}}}{M_{\mathcal{U}}^{d_{U V}-2}}|H|^{2} \mathcal{O}_{I R}
$$

In the following we will assume that $\mathcal{O}_{U V}$ and $\mathcal{O}_{I R}$ are the same operators as in eqns. (2.2) and (2.3) respectively. The dimension of $\mathcal{O}_{I R}$ is usually assumed to lie between 1 and 2 [10], 
as is indeed the case for SQCD. For such operators, this coupling is relevant in the CFT and can significantly change the low energy physics of the unparticle sector. We note there is no symmetry that can forbid this operator without simultaneously forbidding fermion and gauge boson operators coupling to the unparticle sector.

We note that if there is no scalar operator of dimension less than 2 in the unparticle sector, then the operator (3.2) is irrelevant. Any operator with dimension less than 2 would then have to be a vector or higher tensor operator. Such scenarios are difficult to realize in SQCD, but may be realized in more exotic theories. (For example, one may use AdS/CFT and consider the CFT dual of an AdS theory which only contains vector fields.) We will not consider this possibility further.

Once the Higgs acquires a vev, the operator (3.2) introduces a scale into the CFT. This relevant operator will cause the unparticle sector to flow away from its conformal fixed point and the theory will become non-conformal at a scale $\Lambda_{\mathcal{L}}$, where

$$
\Lambda_{\mathcal{U}}^{4-d_{\mathcal{U}}}=\left(\frac{\Lambda_{\mathcal{U}}}{M_{\mathcal{U}}}\right)^{d_{U V}-d_{\mathcal{U}}} M_{\mathcal{U}}^{2-d_{\mathcal{U}}} v^{2} .
$$

Below this scale the unparticle sector presumably becomes a traditional particle sector. For consistency we require $\Lambda_{\mathcal{H}}<\Lambda_{\mathcal{U}}$. If there is to be any sense in which the theory is truly conformal the two scales should be well separated.

Breaking of the conformal invariance due to the new operators has important implications for unparticle phenomenology. For any given experiment, unparticle physics will only be relevant if

$$
\Lambda_{\mathcal{H}}<Q
$$

where $Q$ is the typical energy of the experiment. For lower energies, the unparticle sector can be treated as a particle sector. The constraint of (3.4) then takes the form

$$
Q^{4-d_{\mathcal{U}}}>\left(\frac{\Lambda_{\mathcal{U}}}{M_{\mathcal{U}}}\right)^{d_{U V}-d_{\mathcal{U}}} M_{\mathcal{U}}^{2-d_{\mathcal{U}}} v^{2} .
$$

This suggests that low energy experiments may not be sensitive to unparticle physics. To see this explicitly we note that any observable effect of the operator (2.3) will be proportional to

$$
\epsilon=\left(\frac{\Lambda_{\mathcal{U}}}{M_{\mathcal{U}}}\right)^{2 d_{U V}-2 d_{\mathcal{U}}}\left(\frac{Q}{M_{\mathcal{U}}}\right)^{2\left(d_{\mathcal{U}}+l-4\right)}
$$

Then the effects of the unparticle sector on observables are bounded by

$$
\epsilon<\left(\frac{Q}{M_{\mathcal{U}}}\right)^{2 l}\left(\frac{M_{\mathcal{U}}}{v}\right)^{4} .
$$

It is interesting that this constraint is completely independent ${ }^{1}$ of both the UV and IR scaling dimension of the CFT operator and the potential effects of the unparticle sector are

\footnotetext{
${ }^{1}$ This is not entirely true as there is dependence on $d_{\mathcal{U}}$ due to the modification of phase space as well as dimensionless couplings in the Lagrangian. This results in corrections of order 1, but the dependence on energy scales remains the same.
} 
constrained by only 3 parameters: the experimental energy, scale of the electroweak symmetry breaking and the energy scale at which the interactions between the SM and unparticle sector are generated.

Let us now concentrate on effects on (g-2), the anomalous magnetic moment of the electron. In this case the SM operator is $\operatorname{simply}^{2} \mathcal{O}_{S M}=\bar{e} e$ and the relevant energy scale is $m_{e}$. Therefore eqn. (3.7) becomes

$$
\epsilon<\frac{m_{e}^{6}}{M_{\mathcal{U}}^{2} v^{4}} .
$$

Since effective field theory works at the electroweak scale, we expect $M_{\mathcal{U}} \gtrsim 100 \mathrm{GeV}$. We then find $\epsilon<10^{-28}$. This should be contrasted with the existing experimental constraint given in $[15], \epsilon<10^{-11}$. Therefore the effects of the unparticle sector are completely invisible in (g-2) experiments.

It is clear from (3.6) that signals of unparticle physics increase with energy and the LHC is the most promising place where unparticle physics can be discovered. For such an experimental discovery to be possible, $M_{\mathcal{U}}$ should not be too high. Assuming that we can detect deviations from the Standard Model of order $\epsilon \sim 1 \%$, we see that unparticle physics will be visible at the $\mathrm{LHC}$ as long as $M_{\mathcal{U}} \simeq 10^{5} \mathrm{GeV}$.

If SQCD is the model of the unparticle sector $d_{U V}$ is either 1 or 2. Using (3.3) we can easily see that the case of $d_{U V}=1$ typically predicts breaking scales well above energies probed at the LHC making unparticles irrelevant even there. However, this particular coupling may not be present or there may exist other realizations of unparticles in which $d_{U V}=1$ is not allowed, in which case it is still possible to be at the fixed point for LHC energies. In Figure 1 we show how the breaking scale $\Lambda_{\mathcal{U}}$ varies with $d_{\mathcal{U}}$ and $\Lambda_{\mathcal{U}}$ for two different choices of $d_{U V}$; in both cases $M_{\mathcal{U}}=10^{5} \mathrm{GeV}$, although for $d_{U V}=2$ there is no dependence on $M_{\mathcal{U}}$. From these plots we can see that the range over which the unparticle sector is conformal can be made large only at the expense of increasing the UV dimension of the unparticle operator or the scale $M_{\mathcal{U}}$. The former then requires very large anomalous dimensions to allow $d_{\mathcal{U}}$ to lie between 1 and 2, and the latter may make the discovery of unparticles beyond the reach of any experiment. On the other hand, as we will see in the next section if $\Lambda_{\mathcal{L}}$ is not very small, then the deviations from both particle and unparticle physics may be measurable.

\section{New effects in non-unparticle physics}

In a pure conformal theory, the correlator can be written as

$$
\left\langle O_{\mathcal{U}}(x) O_{\mathcal{U}}(0)\right\rangle=\int \frac{d^{4} P}{(2 \pi)^{4}} e^{-i p x}\left|\left\langle 0\left|O_{\mathcal{U}}\right| P\right\rangle\right|^{2} \rho\left(P^{2}\right)
$$

with

$$
\left|\left\langle 0\left|O_{\mathcal{U}}\right| P\right\rangle\right|^{2} \rho\left(P^{2}\right)=A_{d_{\mathcal{U}}} \theta\left(P^{0}\right) \theta\left(P^{2}\right)\left(P^{2}\right)^{d_{\mathcal{U}}-2}
$$

\footnotetext{
${ }^{2}$ There is also the possibility of a pseudoscalar operator $\bar{e} \gamma_{5} e$ but this follows the same scaling arguments.
} 

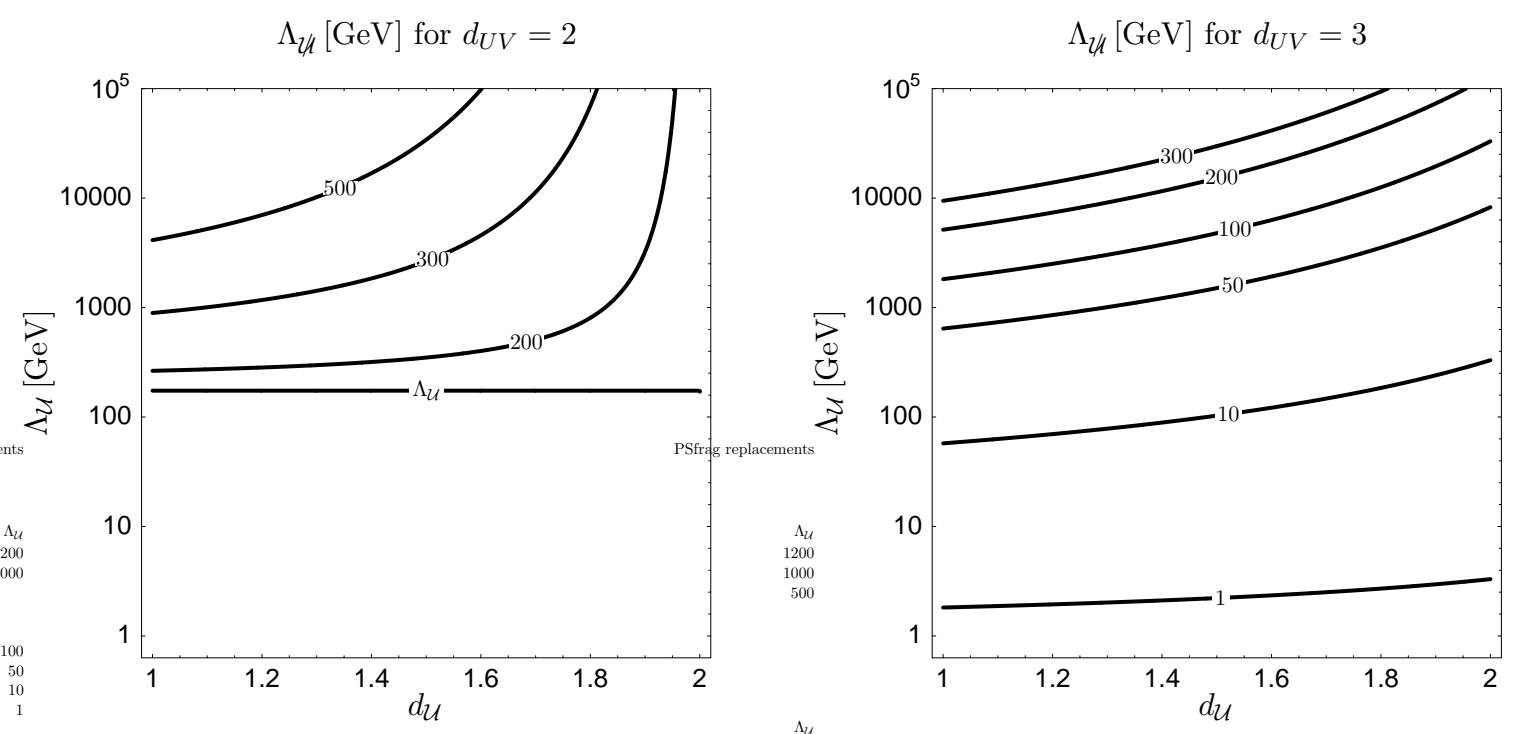

Figure 1: Contours of fixed CFT breaking scale, $\Lambda_{\mathcal{L}}$, as a function of the IR dimension of the unparticle operator, $d_{\mathcal{U}}$, and the scale at which it becomes conformal, $\Lambda_{\mathcal{U}}$. Two particular choices for the UV dimension of the unparticle operator are made, $d_{U V}=2,3$. As discussed in the text we only consider $\Lambda_{\mathcal{H}} \leq \Lambda_{\mathcal{U}}$.

We propose a simple toy model where conformal invariance is broken at a low energy $\mu$ by modifying the above equation to

$$
\left|\left\langle 0\left|O_{\mathcal{U}}\right| P\right\rangle\right|^{2} \rho\left(P^{2}\right)=A_{d_{\mathcal{U}}} \theta\left(P^{0}\right) \theta\left(P^{2}-\mu^{2}\right)\left(P^{2}-\mu^{2}\right)^{d_{\mathcal{U}}-2}
$$

This modification corresponds to shifting the spectrum to remove modes with energy less than $\mu$.

This model maintains the unparticle nature of the hidden sector while including the effects of the breaking of scale invariance. While it is not clear whether such a modification can arise from a consistent QFT, this represents a simple model to study effects of deviations from conformal invariance. More generally, once scale invariance is broken, there may be particle-like modes that would appear as isolated poles in the spectral function; we ignore these effects in this simple model. It would be interesting to see if consistent models of this type can be constructed and what features they possess ${ }^{3}$.

This modification can produce observable effects. To illustrate this, we will reconsider the effects of unparticle physics on the decay of the top through processes like $t \rightarrow u \mathcal{O}_{\mathcal{U}}$. The decay rate for this process can be computed following [9] to be

$$
m_{t} \frac{d \log \Gamma}{d E_{u}}=4 d_{\mathcal{U}}\left(d_{\mathcal{U}}^{2}-1\right)\left(\frac{m_{t}}{M}\right)^{6}\left(\frac{E_{u}}{m_{t}}\right)^{2}\left(1-2\left(\frac{m_{t}}{M}\right)^{2} \frac{E_{u}}{m_{t}}\right)^{d_{\mathcal{U}}-2}
$$

\footnotetext{
${ }^{3}$ We thank C. Csaki and H. Georgi for emphasizing this point to us.
} 


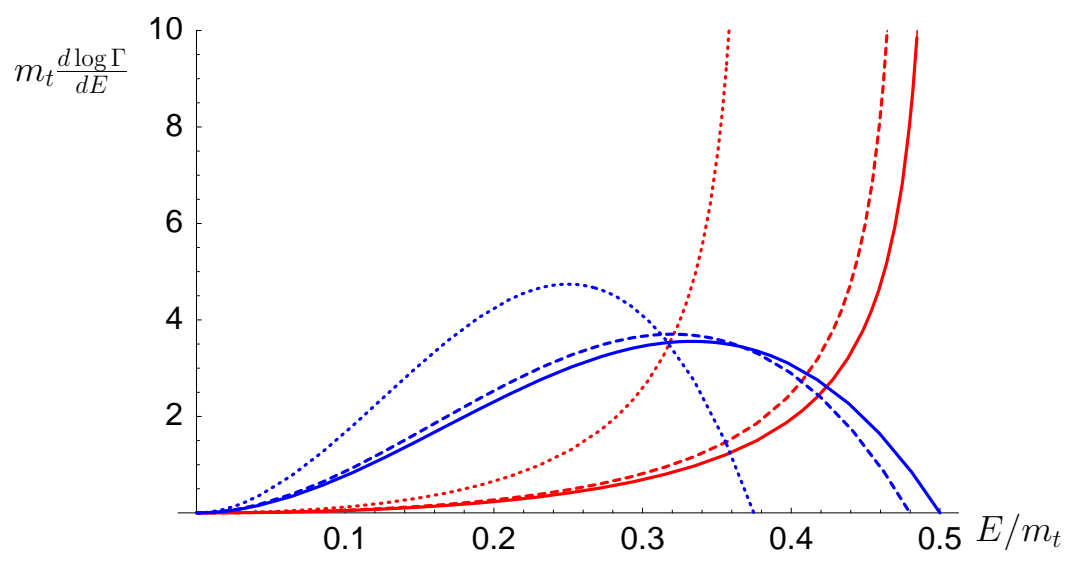

Figure 2: The differential decay rate, $m_{t} \frac{d \log \Gamma}{d E_{u}}$, for the decay $t \rightarrow u \mathcal{O}_{\mathcal{U}}$ as a function of final state quark energy. The red curves (concave) assume the IR dimension of the unparticle operator is $d_{\mathcal{U}}=4 / 3$ and the blue (convex) assume $d_{\mathcal{U}}=3$. In both cases the solid, dashed and dot-dashed curves label the scale, $\mu$, below which there are no unparticle modes and correspond to $\mu / m_{t}=0,0.2,0.5$ respectively.

with $M^{2}=m_{t}^{2}-\mu^{2}$. In Figure 2 we show this modification for various choices of $\mu$ and $d_{\mathcal{U}}$. Notice that the end point of the distribution is no longer $m_{t} / 2$ but is now $\frac{m_{t}^{2}-\mu^{2}}{2 m_{t}}$ and that the normalization of the distribution changes.

It may also be possible to look for effects of the breaking of scale invariance in other collider signatures, for instance the interference between unparticle and SM propagators in simple processes such as $e^{+} e^{-} \rightarrow \mu+\mu^{-}$[10]. It would be especially interesting to see how the inclusion of the coupling between the SM Higgs and the unparticle sector affects Higgs physics. These questions are left for future work.

\section{Acknowledgements}

We thank Howard Georgi for comments on the manuscript. We thank M. Bander for discussions. The work of AR is supported in part by NSF Grant PHY-0354993. PJF was supported in part by the Director, Office of Science, Office of High Energy and Nuclear Physics, Division of High Energy Physics, of the US Department of Energy under contract DE-AC02-05CH11231. PJF would like to thank the Particle Theory group at UC Irvine for hospitality while part of this work was completed.

\section{References}

[1] Y. Liao, Bounds on unparticles couplings to electrons: From electron g-2 to positronium decays, arXiv:0705.0837 [hep-ph].

[2] T. M. Aliev, A. S. Cornell, and N. Gaur, Lepton flavour violation in unparticle physics, arXiv:0705.1326 [hep-ph]. 
[3] G.-J. Ding and M.-L. Yan, Unparticle physics in dis, arXiv:0705.0794 [hep-ph].

[4] C.-H. Chen and C.-Q. Geng, Unparticle physics on cp violation, arXiv:0705.0689 [hep-ph].

[5] M. Luo and G. Zhu, Some phenomenologies of unparticle physics, arXiv:0704.3532 [hep-ph].

[6] K. Cheung, W.-Y. Keung, and T.-C. Yuan, Novel signals in unparticle physics, arXiv:0704.2588 [hep-ph].

[7] X.-Q. Li and Z.-T. Wei, Unparticle physics effects on d-anti-d mixing, arXiv:0705.1821 [hep-ph].

[8] S. Catterall and F. Sannino, Minimal walking on the lattice, arXiv:0705.1664 [hep-lat].

[9] H. Georgi, Unparticle physics, hep-ph/0703260.

[10] H. Georgi, Another odd thing about unparticle physics, arXiv:0704.2457 [hep-ph].

[11] N. Seiberg, Exact results on the space of vacua of four-dimensional susy gauge theories, Phys. Rev. D49 (1994) 6857-6863, hep-th/9402044].

[12] N. Seiberg, Electric - magnetic duality in supersymmetric nonabelian gauge theories, Nucl. Phys. B435 (1995) 129-146, hep-th/9411149.

[13] K. A. Intriligator and N. Seiberg, Lectures on supersymmetric gauge theories and electricmagnetic duality, Nucl. Phys. Proc. Suppl. 45BC (1996) 1-28, hep-th/9509066.

[14] T. Banks and A. Zaks, On the phase structure of vector-like gauge theories with massless fermions, Nucl. Phys. B196 (1982) 189.

[15] G. Gabrielse, D. Hanneke, T. Kinoshita, M. Nio, and B. Odom, New determination of the fine structure constant from the electron g value and qed, Phys. Rev. Lett. 97 (2006) 030802. 